\title{
seal of approval program for radio and television
}

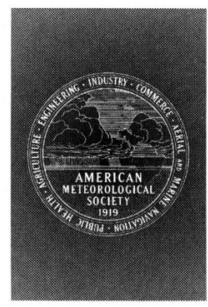

\section{THE PROGRAM AS OF $1997^{1}$}

The goal of the Seal of Approval program is to continuously upgrade radio and television weather programs. Since the weathercaster is the primary representative of the meteorological profession to the public, we, as meteorologists, have a responsibility to help recognize those who are competent. The Board of Broadcast Meteorology was formed by the Council in 1957 to establish criteria for the evaluation of weathercasters and to conduct the evaluation procedure. The growth of television and radio has been accompanied by an increasing impact on the public; therefore, the need for professional weathercasting and its recognition by the AMS has been quite apparent. Evaluation procedures were established and the first television kinescope recording and radio tapes were evaluated by the Board in late 1959. The first Seals of Approval, other than for Board members, were granted in January 1960. Seals have been awarded to weathercasters with widely varied approaches to presenting the weather on television. To date, over 900 Television and over 150 Radio Seals of Approval have been awarded. It has always been the goal of the Seal of Approval program to foster high standards of professionalism among broadcast meteorologists. Recently, however, the courts have attacked the use of the term "professionalism" as being overbroad and capable of misuse. Indeed, this standard has many different meanings to different individuals. We have produced a more specific definition. Henceforth, the stated goals of the program will be to ensure that meteorologists who hold the Seal of Approval exhibit scientific competence and effective communication skills in their weather forecasts.

\footnotetext{
'The Board is currently made up of Bryan Busby, KMBC-TV, Kansas City, MO, chairperson; Vincent D. Condella, WITI-TV, Milwaukee, WI; Frederick J. Gadomski, The Pennsylvania State University, University Park, PA; Tammy A. Garrison, WDRBTV, Louisville, KY; Michael H. Graham, WAFB-TV, Baton Rouge, LA; Roy L. Leep, WTVT-TV, Tampa, FL; Tim B. McGill, Chicagoland TV, Oak Brook, IL; David M. Miller, WFRV-TV, Green Bay, WI; and Donald J. Paul, WIVB-TV, Buffalo, NY.
}

\section{APPLICATIONS}

A. Upon request from any individual, the Secretary-Treasurer of the Society will furnish an application form. Applications will be accepted only from full Members of the AMS or those non-Members who meet all qualifications for full Member as defined in the Constitution of the Society (see also Section VI.E).

Members admitted under Article III, Section 4.B of the Constitution will also need at least 12 semester hours of study in the atmospheric, oceanic, or related hydrologic sciences from an accredited institution of higher learning. At least 8 of the 12 semester hours, with a minimum of two credits in each of three of the following five areas, is required:

1) atmospheric or oceanographic dynamics;

2) atmospheric or oceanographic thermodynamics;

3) physical meteorology or oceanography;

4) synoptic meteorology (or weather systems) or synoptic oceanography;

5) hydrology.

The core courses must include basic processes relevant to atmospheric or oceanic systems. (A memorandum concerning the above requirements was published as an AMS Notice in the June 1991 issue of the Bulletin.)

B. The application form must be completed and returned to the Secretary-Treasurer along with the appropriate application fee. The fee for television is $\$ 275$ for Members and $\$ 600$ for non-Members, which includes a subscription to the Bulletin. The fee for radio is $\$ 225$ for Members and $\$ 500$ for non-Members, which includes a subscription to the Bulletin.

C. The Secretary-Treasurer then forwards the application to the Chairperson of the Board of Broadcast Meteorology.

\section{SELECTION OF EVALUATING COMMITTEE}

A. The Board Chairperson will select five members of the Board, who will constitute the reviewing panel for that 
applicant. Each member of the panel shall certify to the Chairperson that he or she is aware of no conflict of interest in accepting the appointment to review any particular applicant. The applicant will be notified in writing of the names and occupations of the members selected and will be given an opportunity to object to any of the Board members selected. An applicant shall be required to state in writing the reasons for any objection to any member of the panel. The Chairperson may either accept the objection from the applicant or refer the question to the Chairperson of the Commission on Professional Affairs for final decision; the Commissioner shall act on the preponderance of the evidence. All applicants shall be notified that unless objection is received within 15 days, the Chairperson will assume that the selected Board members are satisfactory to the applicant and will proceed to the next step.

\section{SUBMISSION OF EVALUATION MATERIALS}

The Chairperson will then request that the applicant submit three video- or audiotape examples of his or her work. These examples must be of broadcasts made on three consecutive appearance days. In the case of individuals currently employed "on-air," the tapes shall be of actual onair performances, including lead-ins and throwbacks to other on-air talent. All commercial messages shall be edited out of the tape. No minimum time of each segment will be required. Those not currently employed are allowed to submit equivalent simulated tapes. Performances on tapes submitted must be less than six months old as of the date of the application.Applicants must also certify that weathercasts submitted are substantially the result of their own work.

\section{GRADING PROCESS}

A. The applicant sends a copy of his or her tapes directly to each member of the reviewing panel. The Board will review the tapes based on four criteria:

\section{Technical competence}

This is a two-step evaluation. First, all candidates must meet the requirements outlined above in Section I.A or, if not a Member of the Society, demonstrate sufficient educational and professional background to meet these standards for Seal of Approval applicants. The second step is an evaluation of the submitted tapes to determine whether the information contained therein is scientifically and technically valid.

\section{Informational value}

This category will be used to evaluate whether the candidate has given the audience sufficient information about recent, current, and anticipated weather conditions locally and nationally.

\section{Explanatory value}

Reviewers are asked to determine whether or not the candidate has given sufficient explanation of the processes that produce the recent, current, and anticipated weather conditions.

\section{Communication skills}

This criterion is intended to measure the effectiveness of the means chosen by the candidate to communicate information and explanations. Since this is a highly subjective area, only a candidate's clear failure to communicate the information will be judged as grounds for failure. Subjective judgments concerning appearance, sets, lead-ins, props, map symbols, and voice quality will not be used unless cumulatively the negative effect of a poor performance in any of these areas compels the conclusion that the candidate has completely failed to communicate his or her message.

B. Grades will be awarded in each of the four categories on a scale of 4.0, as follows:

1.0: unacceptable

2.0: substandard

2.5: satisfactory (passing)

3.0: good

3.5: excellent

4.0: outstanding

Each reviewer will submit a grade in each category. The grades given by all members will be averaged by the Chairperson. A candidate must score at least a 2.5 average in each category in order to succeed on the examination.

\section{NOTIFICATION}

A. Successful applicants will be notified by letter. In addition, they will be sent a press release from Society headquarters for possible use.

B. Unsuccessful applicants will be notified by letter from the Secretary-Treasurer of the Society. The letter shall contain a statement from the Chairperson of the Commission, explaining the failure and suggesting ways of improving the performance based upon the information received from Board members. Unsuccessful ap- 
plicants may reapply after a period of three months from the date of the negative notification. The reapplication fee is $\$ 100$. After a second unsuccessful application, there will be a one-year waiting period before a third application can be submitted. However, if more than two (2) years have passed from the date of the negative notification on the applicant's initial application, then the second application shall be deemed a new application.

\section{MISCELLANEOUS}

A. Successful candidates may move their Seal of Approval from station to station without further application to the Society. Relocation from a national (for example, $\mathrm{CNN}$ or The Weather Channel) to a local appearance, however, requires the submission of new tapes (but no additional fee) for review by the Board in accordance with the four stated criteria.

B. Seals may be renewed annually by submitting a renewal fee and attesting that the overall content and format of on-air presentations have not changed significantly. The annual renewal fee for Members is $\$ 20$, and for non-Members, $\$ 100$.

C. Successful candidates will be informed both of their right to use the Seal of Approval and of any limitations that the Society may impose upon such use.

D. Unsuccessful candidates may appeal the negative decision of the Board of Broadcast Meteorology to the Executive Committee of the Society within 90 days of the date of notification. The fee for an appeal is $\$ 100$; however, the Executive Committee reserves the right to waive the fee in cases of financial hardship.

E. All candidates and reapplicants must meet the current Member (see "Modifications to Policies Governing the Seal of Approval Program" and "Interpretive Memorandum," Bull. Amer. Meteor. Soc., 70, 636-637; and AMS Notice, Bull. Amer. Meteor. Soc., 70, 837) and seal applicant criteria at the time of their seal application or reapplication form, as outlined in Section I.A.

\section{SUSPENSION OR REVOCATION}

A. The AMS reserves the right to suspend or revoke the right to use the Seal of Approval if a sealholder, in the conduct of his or her profession, clearly fails to conduct himself or herself in a manner that reflects the dignity and honor of the profession or if a sealholder fails repeatedly to adhere to the four criteria for the award of the seal set out in Section IV.
B. Any complaint that may be grounds for suspension or revocation of the Seal under Paragraph A shall be sent to the Secretary-Treasurer of the Society. The Secretary-Treasurer may summarily dismiss a frivolous complaint with notice and a statement of reasons to the complainant. Otherwise, the Secretary-Treasurer shall send a copy of the complaint to the Chairperson of the Commission on Professional Affairs, the Chairperson of the Board of Broadcast Meteorology, and the sealholder.

C. The Chairperson of the Board of Broadcast Meteorology may resolve any complaint by any appropriate informal means, which may include contacting the sealholder in question in an effort to resolve by mutual agreement the subject of the complaint.

D. Any complaint not disposed of by informal means shall be determined as follows:

1. With the concurrence of the Chairperson of the Commission on Professional Affairs, a copy of the complaint shall be sent to the sealholder in question by the Board Chairperson, together with a copy of Section VII constituting the procedure for suspension or revocation of the Seal of Approval and the names and brief descriptions of the members of a Fact-Finding Panel appointed under Sub-Paragraph 2.

2. The Board Chairperson shall select three members of the Board, who will constitute a Fact-Finding Panel to determine the issues raised in the complaint. Each member of the Fact-Finding Panel shall certify to the Chairperson that he or she is aware of no conflict of interest in accepting the appointment to the Fact-Finding Panel. The sealholder in question shall have the opportunity within 15 days of the date of notification to object to any member of the Fact-Finding Panel. The sealholder shall be required to state in writing the reasons for any objection to a member of the panel. The Chairperson may either accept the objection from the sealholder or refer the question to the Chairperson of the Commission on Professional Affairs for final decision.

3. The sealholder shall cooperate fully with the FactFinding Panel and produce any tangible information relevant to the issues raised in the complaint and shall attempt to secure for the Fact-Finding Panel copies or recordings of any weathercast in issue. The sealholder may submit to the Fact-Finding Panel any other information he or she deems relevant, including copies or recordings of other weathercasts and a written answer to the complaint. 
4. After the submission of the tangible evidence to the Fact-Finding Panel, if any facts remain in dispute, a hearing shall, upon written request of the sealholder, be held by the Fact-Finding Panel upon adequate notification to the sealholder and at a time and place convenient to the members of the panel and the sealholder. The hearing shall be conducted according to fundamental concepts of due process recognized as fair and followed by administrative agencies in the conduct of adjudicatory hearings, which shall include the right to counsel, presentation of witnesses, right to cross-examination, and the right to record the proceeding by either stenographic or tape-recording means. Strict rules of evidence shall not apply, but the panel shall accept information or evidence that is customarily relied upon by reasonable people in the conduct of serious affairs.

5. The Fact-Finding Panel shall make written findings of fact and shall determine if the sealholder has, in the conduct of his or her profession, clearly failed to conduct himself or herself in a manner that reflects the dignity and honor of the profession, or if the sealholder has failed repeatedly to adhere to the four criteria for the award of the Seal of Approval as set out in Section IV.

If the Fact-Finding Panel determines that the sealholder has failed in his conduct or adherence to the criteria as aforesaid, then the Fact-Finding Panel shall include in its written decision its findings on the degree of severity of the matter and a recommendation for the imposition of sanctions, which may include:

a. a written informal admonition by the Chairperson of the Commission on Professional Affairs, a copy of which shall be retained in the sealholder's personal file; or

b. a suspension of the sealholder's right to use the Seal of Approval for a period of time; or

c. a revocation of the sealholder's Seal of Approval.

6. The written decision of the Fact-Finding Panel shall be sent to the Chairperson of the Board of Broadcast Meteorology, the Chairperson of the Commission on Professional Affairs, and the sealholder. The Chairperson of the Board of Broadcast Meteorology and the Chairperson of the Commission on Professional Affairs, after a review of the decision, shall jointly determine in their judgment the appropriate sanction and administer the same.

E. A sealholder against whom action has been taken may, within 30 days of the date of notification of the action, appeal the action in writing to the Executive Committee of the Society (see Organizational Procedures of the AMS).

$\begin{array}{lr}\text { Abrams, Elliot } & 51 \\ \text { Allen, Mark } & 143 \\ \text { Arellano, Michael J. } & 151 \\ \text { Babikian, Gary } & 117 \\ \text { Bridgers, George M. } & 154 \\ \text { Boyle, John L. } & 139 \\ \text { Cosgrove, Lawrence F. } & 137 \\ \text { Dahl, David W. } & 101 \\ \text { Davis, Francis K., Jr. } & 1 \\ \text { Eichorn, David N. } & 119 \\ \text { Endersen, G. William } & 90 \\ \text { Eustis, Allan C. } & 56 \\ \text { Falconer, Raymond E. } & 9 \\ \text { Fathauer, Theodore F. } & 140 \\ \text { Fidler, James C. } & 13 \\ \text { Filloon, Karen A. } & 103 \\ \text { Finckle, Earl S. } & 50 \\ \text { Fishel, Gregory B. } & 120 \\ \text { Geyer, James R. } & 97 \\ \text { Glickman, Todd S. } & 71 \\ \text { Goldbaum, David A. } & 145 \\ \text { Head, Paul A. } & 121\end{array}$

Heller, Kathleen

Hodges, Dennis J.

Horstmeyer, Steven L.

Jacquemin, William G.

Jaggers, James W.

Joseph, Paul S

Kimmel, Troy M., Jr.

Kinnan, Henry W.

Koplien, Craig S.

Landin, Michael G.

LaPoint, Michael S

Madson, Michael J.

Mahar, Wayne G.

Maly, Daniel D.

McKinley, Kenneth C.

McLain, Robert W.

Melhuish, Kirk

Mercer, Jack M.

Mlodzik, Joseph R.

Moldenhauer, Donald M.

Muzio, Miles S.
89

147

76

127

123

46

110

3

138

136

153

113

114

63

107

43

141

68

135

130

108
Newman, Steven B.

126

Norman, Royal L., Jr.

Oldshue, John H.

Peterson, William D.

Phillips, Ed

Prater, Judson E., Jr.

Randby, William E.

Rao, Joseph

Rao, Renate

Rubin, Eugene M.

Schulz, Kenneth A.

Shaw, Scott A.

Shutt, Charles T.

Spencer, Lisa L.

Stephan, George F.

St. Pe, Edward I.

Toohey-Morales, John

Wahl, Jillene A.

Weiner, Craig A.

Williams, Kevin D.

Wright, George M.
112

150

72

79

142

109

118

115

73

111

152

124

144

133

128

146

148

106

93

131 


\begin{tabular}{|c|c|c|}
\hline 1 & Francis K. Davis, Jr. (H) & 1959 \\
\hline 2 & Kenneth H. Jehn* $(\mathrm{H})$ & 1959 \\
\hline 3 & Henry W. Kinnan (H) & \\
\hline 4 & Milton F. Barlow** & \\
\hline 5 & Albert E. Boyer, Jr.** & 60 \\
\hline 6 & Robert L. Hendrick** & \\
\hline 7 & George M. Howe** & \\
\hline 8 & James M. Macdonald, Jr.** & \\
\hline 9 & Frederick P. Ostby, Jr.** & \\
\hline 10 & Howard M. Frazier** & \\
\hline & Robert G. Beebe** & \\
\hline 2 & Howard H. Hanks, Jr.* & \\
\hline 13 & James C. Fidler $(\mathrm{H})$ & \\
\hline 14 & Lawrence R. Mahar* & \\
\hline 15 & Eugene C. Grueber** & \\
\hline 16 & Norman F. Lacey* & \\
\hline 17 & Alan F. Jones** & \\
\hline 18 & Conrad B. Gosset** & \\
\hline 19 & Raymond E. Falconer & \\
\hline 20 & Albert W. Duckworth** & \\
\hline 21 & William J. Hartnett* & \\
\hline 22 & J. R. Dixon* & \\
\hline 23 & Armand R. Iaccheo** & \\
\hline 24 & Stuart P. Marr** & \\
\hline 25 & John A. Ebert** & \\
\hline 26 & Ray A. Cathcart** & \\
\hline 27 & Harry C. Zimmerman** & \\
\hline 28 & Thomas W. Spence** & \\
\hline 29 & John W. Hambleton** & \\
\hline 30 & Seth D. Kemble** & \\
\hline 31 & John H. Erikson** & \\
\hline 32 & Kenneth C. Garee** & \\
\hline 33 & Robert F. Zames** & 1967 \\
\hline 34 & William B. Hovey** & 196 \\
\hline 35 & Conrad L. Johnson** & \\
\hline 36 & Fred Norman** & 67 \\
\hline 37 & Leonard M. Slesick** & $196^{\prime}$ \\
\hline 38 & Walter Devanas** & $196^{7}$ \\
\hline 39 & Jehu D. Ashmore** & 196 \\
\hline 40 & John T. Ghiorse, Jr.** & \\
\hline 41 & Charles M. Umpenhour** & 196 \\
\hline 42 & Sidney O. Barnard** & 197 \\
\hline 43 & Robert W. McLain & 1970 \\
\hline 44 & Thomas G. Wills** & 197 \\
\hline 45 & Walter A. Lyons** & \\
\hline 46 & Paul S. Joseph & \\
\hline 47 & William N. Seiler** & \\
\hline 48 & W. Paul Catoe** & 197 \\
\hline 49 & George R. Wooten, Jr.** & 197 \\
\hline 50 & Earl S. Finckle & \\
\hline-7 & Elliot Abrams & \\
\hline
\end{tabular}

52 Joel N. Myers**

53 George N. Brancato**

54 Robert K. Ryan**

55 Lyle E. Brosche**

56 Allan C. Eustis

57 J. Michael Fritsch**

58 John F. Henz**

59 Vincent R. Scheetz**

60 Virginia J. Bigler-Engler**

61 John T. McMurray**

62 Malcolm P. Sillars**

63 Daniel D. Maly

64 Steven D. Hilberg**

65 Steven A. Root**

66 John R. Stansfield**

67 Todd Gross**

68 Jack M. Mercer

69 Craig A. Johnson**

70 Charles M. Gertz**

71 Todd S. Glickman

72 William D. Peterson

73 Eugene M. Rubin

74 Raymond J. Ban**

75 Gerard K. Butch**

76 Steven L. Horstmeyer

77 William O. Alexander**

78 Howard K. Bryant**

79 Edwin Phillips

80 Aaron Williams, Jr.**

81 Joseph A. Dandrea**

82 Thomas L. Magnuson**

83 James H. Block**

84 Beverely S. Cudbird*

85 Donald L. Edwards***

86 Gregory J. Story**

87 John C. Dooley**

88 Daniel F. Smith**

89 Kathleen Heller

90 G. William Endersen

91 Ronald W. Trenda**

92 Michael W. Lynch**

93 Kevin D. Williams

94 Lee C. Anderson**

95 Jim F. Riggs*

96 Lloyd G. Tidwell**

97 James R. Geyer

98 Jeffrey B. Lawson**

99 Brian W. Jarvis**

100 Timothy A. Legel**

101 David W. Dahl

102 Sharon L. Akemann**
1973

1973

1973

1974

1974

1976

1976

1976

1976

1977

1977

1978

1978

1978

1978

1979

1979

1979

1979

1979

1979

1980

1980

1980

1980

1981

1981

1981

1981

1981

1981

1981

1981

1981

1981

1982

1982

1982

1982

1982

1982

1982

1982

1982

1983

1983

1983

1983

1983

1983

1983
103 Karen A. Filloon

104 Michael L. McCallum**

105 Timothy K. Ecker**

106 Craig A. Weiner

107 Kenneth C. McKinley

108 Miles S. Muzio

109 William E. Randby

110 Troy M. Kimmel, Jr.

111 Kenneth A. Schulz

112 Royal L. Norman, Jr.

113 Michael J. Madson

114 Wayne G. Mahar

115 Renate M. Rao

116 William S. Schmidt**

117 Gary Babikian

118 Joseph M. Rao

119 David N. Eichorn

120 Gregory B. Fishel

121 Paul A. Head

123 James W. Jaggers

124 Charles T. Shutt

125 Peter V. Chan**

126 Steven B. Newman

127 William G. Jacquemin

128 Edward I. St. Pe

129 Harold S. Austin, Jr.**

130 Donald M. Moldenhauer

131 George M. Wright

132 Phillip W. Badgett**

133 George F. Stephan

134 Geoffrey P. Eden**

135 Joseph R. Mlodzik

136 Michael G. Landin

137 Lawrence F. Cosgrove

138 Craig S. Koplien

139 John L. Boyle

140 Theodore F. Fathauer

141 Kirk T. Melhuish

142 Judson E. Prater, Jr.

143 Mark Allen

144 Lisa L. Spencer

145 David A. Goldbaum

146 John Toohey-Morales

147 Dennis J. Hodges

148 A. Jillene Wahl

149 Kevin L. Orpurt**

150 John H. Oldshue

151 Michael J. Arellano

152 Scott A. Shaw

153 Michael S. LaPoint

154 George M. Bridgers
1983

1983

1985

1985

1985

1985

1985

1985

1986

1986

1986

1987

1987

1987

1987

1987

1987

1987

1987

1988

1988

1988

1988

1988

1988

1987

1989

1989

1989

1989

1989

1989

1990

1990

1991

1992

1992

1992

1992

1992

1992

1993

1993

1993

1994

1994

1994

1994

1994

1995

1997

$(\mathrm{H})=$ Honorary

*Deceased **Inactive 
Abrams, Elliot

Adams, John W.

Adams, Kimberly

Adamson, Steve

Addis, Richard A.

Adrian, Barton J.

Aguilera, David W.

Akemann, Sharon L.

Albert, Richard E.

Albrecht, Brian A.

Alger, Michael P.

Allen, Mark

Allen, Ron

Alworth, Brian T.

Amble, Gary J.

Andersen, Adolph M., Jr.

Anderson, Mike S.

Annen, William H.

Arnold, Robert

Arredondo, Carl C., III

Atkins, Bret Martin

Atkinson, Daniel M., Jr.

Aucoin, Kenneth A.

Austin, Martha L.

Avalos, Andrew A.

Avery, Bruce M.

Aydelott, James D.

Baden, Mark J.

Baker, David

Bakula, William R.

Barach, Jim

Barlow, Kenneth J.

Barnes, David P.

Barnhill, Fredrick R.

Baron, Robert $\mathrm{O}$.

Barone, Dianne

Barron, Cory

Bartlett, Joel P., Jr.

Barton, Neal, Jr.

Barys, Paul E.

Becker, Robert

Beckman, John F.

Behm, Gordon R.

Belau, Matthew P.

Bell, Charles G.

Bellas, Stephen C.

Bennett, Terri L.

Berkowitz, Zachary R.

Bernard, David

Bernier, Andre M.

Bernier, John G.

Bernier, Sally $\mathrm{P}$.

Bernstein, Howard I.

Bickford, Daniel

Bielski, John T.

Billingsley, Franklin P.

Blalock, Scott D.
82

410

914

831

255

303

871

429

112

404

618

792

360

460

541

749

542

233

332

551

614

203

463

449

407

604

775

768

848

814

771

533

353

532

281

666

765

140

832

137

262

100

444

424

685

588

680

681

890

286

274

319

615

816

287

596

737
Bogner, Mark L.

Bohnak, Karroll R.

Bolton, Albert M.

Boney, Stan

Bono, Michael

Bosley, James W.

Boss, William H.

Bouchereau, Paul E., Jr.

Boylan, Raymond L.

Boyle, John L.

Bracciano, Michael J.

Bradley, Chris

Brandt, Barry A.

Bray, Steven C.

Brookins, Michael A.

Brough, Robert C.

Brown, David H.

Brown, David W.

Brown, George G.

Brown, Patricia M.

Brown, Scott

Browne, Steven W.

Browning, Wes

Brunet, Rene J., III

Burbank, Barry M.

Buresh, Michael A.

Burns, Glenn N.

Burse, Tom

Busby, Bryan T.

Buser, Donald C.

Calbreath, Joseph R.

Caldes, George T.

Calhoun, Joseph J.

Cannalte, Gary A.

Cannon, Declan P.

Caporizzo, Steven P.

Cardosi, Jay A.

Cariello, Frank J.

Carlson, David C.

Carson, Donald W.

Carter, Kevin M.

Cascione, Steven R.

Casey, Terrence P.

Cash, Jonathan R.

Castles, Jed R.

Cavalier, Anthony

Cejka, Michael G.

Cessarich, John A.

Chandik, John F.

Chandley, David I.

Chapman, Nancy R.

Chase, Matthew J.

Childress, Elmer M.

Chisholm, Robin A.

Chisholm, Thomas L.

Chuey, Timothy A.

Cimino, Christopher J.
901

504

307

774

405

239

641

701

139

707

578

722

613

476

817

401

354

759

500

653

884

321

715

451

204

668

259

447

425

310

572

693

338

628

477

390

585

478

349

295

518

462

568

505

748

314

387

457

125

592

244

911

436

739

543

245

724
Cioffi, Joseph

Clark, Noreen R.

Clark, Thomas N.

Clarke, James R.

Coleman, Carey $\mathrm{T}$.

Collins, John J.

Condella, Vincent D., Jr.

Coniglio, Martin E.

Conklin, Alan R.

Connell, Daniel S.

Conway, Joseph C.

Corbin, James M.

Core, Patrick A.

Cosgrove, Lawrence F.

Cousins, Paul W.

Dahl, David W.

Damian, David C.

Daugherty, Walton M.

Davies, Jonathan

Davis, Brian D.

Davis, Francis K., Jr.

Davis, Michael G.

DeBoer, Terri A.

DeBroder, Charles E.

Deegan, Timothy W.

Delkus, Pete W.

Dellegatto, Paul N.

Denardo, Joseph W.

Denton, John V., Jr.

Deprest, Bruce J.

Derda, Anthony $\mathbf{J}$.

DeZubay, Eric T.

Diaz, Joseph R.

Dierks, David G.

DiGiovanni, Joseph V.

Dignon, Nancy E.

Divecchio, Thomas A.

Dixon, Robert W.

Dobrowolski, Daniel G.

Dolan, Linda C.

Dooley, Richard S.

Dorval, Scott C.

Doucet, Gail J.

Duncan, Glen A.

Duncan, James G.

Duranczyk, Edward A., Jr. .

Dvoskin, Norman D.

Edwards, Brad J.

Ehrhardt, Kent

Eichner, Keith

Eichorn, David N.

Eisenson, Robert N.

Eldridge, Evan S.

Elfervig, Leonard R.

Elliot, George

Ellis, Kathy

Epstein, David

339 
Estano, Neal C.

Eubank, Mark E.

Eustis, Allan C.

Evans, Samuel M.

Evans, William H.

Fabrizi, Fulvio A.

Fairbourne, Michael D.

Farley, John A.

Farrell, Christopher M.

Farrell, James T.

Fathauer, Theodore F.

Faurot, Richard W.

Feltgen, Dennis W.

Ferrara, Ellen

Fidler, James C.

Field, Bradford A.

Field, Storm E.

Fields, Ernest G.

Filloon, Karen A.

Finan, Mark

Finden, Roy F.

Fischer, John C.

Fisher, Scott

Fishel, Gregory B.

Fletcher, Richard R.

Flowers, James B.

Fox, Mark A.

Frame, Angelique D.

Frankum, Mark A.

Frary, Dennis G.

Freeman, David

French, Robert

Friedmann, Michael L.

Fries, Todd

Fuchs, Mark C.

Fuller, John A.

Gajownik, Glenn R.

Ganahl, Jym R.

Gandy, James T.

Garcia, Alejandro V.

Garrison, Tammy A.

Gaughan, John C., Jr.

Gelber, Benjamin D.

George, Chuck

George, David L., Jr.

Gerberg, Glen

Gerbstadt, Terry

Geyer, James R.

Ghiorse, John T., Jr.

Giddings, Peter F.

Gile, Bill

Giles, Jimmie D.

Giunta, Mike

Goddard, Richard D.

Goff, Kevin H.

Goldstein, Mel

Gomez, Mario J.

Goosmann, Robert D.

Graham, Michael H.

Graves, Sharon L.
Greenwald, Brian

Gregorius, Robert

Gregory, Nicholas J.

Gregory, Robert E.

Griesgraber, Patrick J.

Griffin, Richard K.

Griffin, Victoria L.

Grigsby, Elhanan P. III

Gross, Paul H.

Gross, Todd

Grote, Christopher R.

Gruner, Elissa L.

Guaraldi, John J.

Guild, David C.

Gurney, Charles E.

Guthrie, Donald T.

Guy, James R.

Hagar, Paul S.

Hale, Thomas E.

Hall, W. Scott

Handley, Lawrence A.

Hardin, Jeffrey G.

Harding, Duane D.

Harman, Donald P.

Harmon, Dave

Harrigan, Robert W.

Harris, John

Harris, Ronald A.

Hartman, David R.

Hartung, Wayne C.

Hauf, Thomas

Hearst, Ronald A., II

Heaton, Jeffrey M.

Hedrick, Timothy R.

Hennen, David A.

Henry, Dan

Heppner, Paul D.

Hernandez, Michael M.

Herring, Charles S.

Higgins, Chris

Hilgartner, John C.

Hill, Jeffrey W.

Hill, Lawrence D.

Hill, Rodney L.

Hinkin, Matthew D.

Hocks, Robert J.

Hodak, Gerald A.

Hoffman, Michael J.

Hohmann, Christopher T.

Hollett, Shane R.

Honore, John C.

Hope, John R.

Hornberger, Art

Horstmeyer, Steven L.

Hosler, Charles

Hovey, William B.

Howes, Ronald L.

Howl, James M.

Huff, Janice W.

Huffines, Bradford T.

Huttner, Paul T.

Jacquemin, William G.

Jaggers, James W.

James, David

Jensen, Belinda

Jensen, Jeffrey T.

Jerve, Steven M.

Johnson, Anthony O.

Johnson, Craig A.

Johnson, Glenn E.

Johnson, Mark E.

Johnson, Mark T.

Johnson, Richard G.

Johnson, Veronica

Johnston, Harry

Jones, Bruce

Jones, Dale L.

Jones, David F.

Jones, Walton W.

Joseph, Paul S.

Kacmarik, Diane

Kaderli, Holice H., Jr.

Kahanek, Kristine

Kalinowski, Bruce E.

Kamal, William D.

Kastor, Neil E.

Katz, Bruce R.

Katzfey, Rick E.

Kearbey, Ric

Keller, Ted W., Jr.

Kelly, Terence F.

Kempf, Warren T.

Keneely, William C.

Kennedy, Arch

Kersh, Stephen W.

Kessler, George H. III

Kierein, Thomas J.

Kimmel, Troy M., Jr.

Kinnan, Henry W.

Kirk, Jeff

Kirschvink, Michael E.

Kirtek, Hugh A., Jr.

Klevanosky, John

Klimasewski, Theodore J.

Klotz, Jeffrey D.

Kniess, Terry L.

Knific, Suzanne S.

Knight, Paul G.

Knight, Thomas

Kolls, Rebecca 
Kronschnabel, Jeffrey F.

Kruhoeffer, Douglas P.

LaNore, Steve C.

LaPoint, Michael S.

LaPointe, Stephen A.

Larson, Mark A.

Latella, Michael J.

Lauria, Joseph V.

Lavine, Matthew A.

Lawrence, Richard J.

Lawson, Jeffrey B.

Lazalier, Jeffrey A.

Lee, Daniel W.

Lee, Mark D.

Leep, Roy L.

Lemanowicz, Kevin P.

Lemke, Cheryl L.

Lessens, George D.

Lewis, Elaine B.

Ley, Gary W.

Lezak, Gary M.

Lindmeier, Robert G.

Lipsey, Suzanne

Little, James R.

Lizura, Joseph

Loffman, Tom

Longley, David P.

Loufman, Jon D.

Lowther, Michael C.

Lozano, Mike A., Jr.

Lucht-Snyder, Lisa

Luehrs, Donald E., Jr.

Luterman, Richard H.

Lutzel, John T.

Lynch, Marcus

Lyons, Jeffrey P.

Lytle, James

Madaus, James L.

Madden, Warren J.

Madson, Michael J.

Maguire, Michael F.

Maguire, Thaddeus J.

Mahar, Wayne G.

Mahoney, Thomas J.

Malan, John J.

Maly, Daniel D.

Mamola, Frank

Mancuso, Mark

Mann, Phillip D.

Marciano, Robert M.

Marquis, Colin N.

Marriott, Richard T.

Marsh, David S.

Marshall, John

Marshment, Roberta A.

Marsik, Frank J.

Martin, Bill

Martin, James H.

Martz, Jerry W.

Mason, Layne P.
Massaro, Mark E.

Mastrostefano, Antonio III

Matthews, Ed C.

Maxon, Bob

Mayo, Scott

McCall, Michael L.

McCauley, Steven D.

McClellan, Michael H.

McCollister, Daryl W.

McCown, Pamela J.

McGill, Tim B.

McLain, Robert W.

McNally, Louis K. III

Meador, Dan P.

Mercer, Jack M.

Meritt, Ron

Meyer, Travis G.

Michaelsen, Clifton W.

Mignone, Anthony R.

Milham, Daniel L.

Miller, David M.

Miller, John M.

Miller, Lori A.

Mitchell, Alan L.

Mitchell, Rick J.

Mlodzik, Joseph R.

Modrick, Michael V.

Moeller, Craig W.

Mogil, H. Michael

Mohr, Samantha

Monahan, Keith R.

Morano, Matthew D.

Moray, Glenn T.

Morelli, Donald F.

Morrison, Cliff

Morrow, Jeffrey T.

Moskowitz, Harvey

Mroz, Paul J.

Mukherjee, Mishtu

Mulholland, Lawrence J.

Mumm, Stephen H.

Murray, David P.

Muzio, Miles S.

Myers, Chad E.

Nefstead, Cyril E.

Nelsen, Mark

Nelson, Michael P.

Nese, Jon M.

Newman, Stephen R

Nichols, James C.

Nicholson, Clifford M.

Nitz, Bradley W.

Noe, Donald S.

Nolan, Warren D.

Norcross, Bryan S.

Norman, Royal L. Jr.

O'Hearn, Michael J.

O'Lenick, Mike

Oliver, David A.

Ollis, Randall D.
Orr, Kathleen A.

Orzel, Brian P.

Ott, Armin J.

Owings, Willis B.

Pann, Anthony J.

Parise, Joseph A.

Parsons, Christopher I.

\section{795}

786

Parsons, Peter G.

550

Pascal, Neal B.

Paul, Donald J.

Pearl, Edward W.

Peitzman, Pamela S.

Penzkowski, Ronald G.

Peppers, Robert W.

Periconi-Balling, Darlene A.

Perillo, Robert

Peterson, William D.

Phillips, Jim

Pietrowicz, Joseph A.

Piotrowski, Edward T.

Pope, Daniel W.

Poulson, Sterling S.

Prater, Judson E., Jr.

Preszler, Cindy

Pringle, H. Benjamin

Prokop, Patrick

Provenzano, Andrew C.

Pyeatt, Janet E.

Race, William P.

Raines, Dallas D.

Raleigh, Stephen

Ramer, Kevan W.

Randby, William E.

Rasor, James I., II

Rector, Thomas C.

Reed, Robin J.

Reh, William G.

Reif, James H.

Reiner, Jeremy

Relihan, David J.

Renner, Jeffrey B.

Resultan, Sharon 
Rubin, Eugene M.

Rubin, Evan A.

Ryan, Robert T.

Sadowsky, Gary

Saeland, Jodi M.

Salna, Erik

Sandusky, Frederick C.

Santoro, David M.

Sanvido, Cecilia

Sater, Tom

Satterfield, Danny E.

Sawyer, David R.

Scalzi, John

Scaman, Thomas S.

Schick, Larry

Schmit, Dan

Schmitz, Kurt R.

Schnebelt, James J.

Schreck, Philip G.

Schubert, William H.

Schulz, Kenneth A.

Schumacher, Anthony T.

Schwartz, Glenn E.

Schwarz, Philip S.

Schwoegler, Bruce W.

Scirto, Mark J.

Sealls, Alan R.

Sebastian, Norman C.

Seidel, Michael P.

Selle, Kevin W.

Settoon, Margaret Orr

Sevcik, David P.

Shapiro, Howard E.

Shattuck, Wayne C.

Shaw, Robert M.

Shaw, Scott A.

Shepard, Stuart A.

Shiels, Robert J.

Shumway, Christopher A.

Shutt, Charles T.

Siebert, D. James

Simon, Mike

Simosko, Ken

Sisto, F. Calvin

Skilling, Thomas E. III

Slater, Jon

Smalley, Jack

Smalstig, Jonathan J.
Smith, Dennis L.

Smith, Jeffrey F.

Smith, Steven W.

536

Smith, Terri A.

Sobel, Joseph P.

Solomon, Bethany

Sonen, Heidi E.

Sopka, Robert J.

Sorrells, Thomas G.

Spakowitz, Bradley J.

Spann, James

Spencer, Lisa L.

Spencer, William R.

Stachak, Stanley J.

Steffen, William J.

Stinnett, Lorraine

Stockhausen, Camie

Stone, Michael W.

St. Pe, Edward I.

Svercl, Stephen J.

Swails, Terry L.

Sweeney, David S.

Swienckowski, Steve

Szymanski, Thomas F.

Taft, Jerome B. II

Tanchak, Jeffrey A.

Tasselmeyer, Thomas M.

Taylor, Reg

Teeling, Steven

Teller, Merril D.

Teske, Robert J.

Textor, Theodore E.

Thomas, Bruce G.

Thomas, Daniel H.

Thompson, Michael L.

Towne, David K.

Tracey, Gerard J.

Travis, Bradley B.

Trenda, Ronald W.

Twigg, Roger M.

Udelson, Stephen M.

Vachalek, Roger E.

Van Dillen, Robert S.

Vanore, Dave

Varga, Theresa L.

Vernon, Connor

Vincent, Samuel J.
Von Ahn, Joan M.

Voss, Valerie

Wagenet, Chris

Wahl, A. Jillene

Walsh, William F.

617

Wankum, Michael D. 549

Wappler, Harry V. 209

Watkins, Mark S. $\quad 769$

Waunsch, John G. $\quad 423$

Weagle, Steve W. $\quad 888$

Weathers, Dwight K. 763

Weaver, Robert 67

Webre, Byron K. $\quad 432$

Weiner, Craig A. $\quad 658$

Weingarten, Andrew L. $\quad 400$

Weiss, Carl 270

Welborn, Jeffrey 659

Wendel, John M. $\quad 317$

Werner, Robert F., Jr. $\quad 512$

Westbrook, Don R. 144

Westerlage, Keith C. 439

Wheeler, John F. $\quad 660$

Whelan, Christopher P. $\quad 803$

Whitelaw, Philip $\quad 312$

Wilkinson, Mark D. $\quad 575$

Williams, Kevin D. $\quad 340$

Williams, Phillip B. 511

Willing, John R. $\quad 232$

Wills, Thomas G. $\quad 80$

Winfield, Alan 419

Winter, John 788

Winterling, George A. $\quad 42$

Wohlenhaus, Stephen J. $\quad 564$

Wood, Glenn C. 271

Woodford, Richard A. $\quad 452$

Woods, Benjamin P. $\quad 594$

Woods, Craig J. 113

Wright, Christopher M. $\quad 689$

Wrobel, Mary E. $\quad 767$

Wysocki, Dean R. $\quad 878$

Yaros, Ronald A. $\quad 272$

Zabrecky, George 431

Zabrecky, Robert E. $\quad 90$

Zaffino, Matthew $\quad 376$

Zandt, Paul A. $\quad 546$

Zimmerman, Glenn $\quad 713$

Zunke, Dale N. 202

\section{TELEVISION SEAL HOLDERS (LISTED BY SEAL NUMBER)}

1 Francis K. Davis, Jr. (H) 1959

2 Kenneth H. Jehn* (H) 1959

3 Henry W. Kinnan (H)

1959

(H) = Honorary

*Deceased **Inactive

10 Roy L. Leep

11 James M. Macdonald, Jr.**

12 Frederick P. Ostby, Jr.**

13 Donald E. Kent**

14 Peter M. Kuhn**

15 Milton A. Strauss**

$\begin{array}{lll}4 & \text { Milton F. Barlow** } & 1960 \\ 5 & \text { Warren A. Culbertson** } & 1960 \\ 6 \text { Bob Thomas** } & 1960 \\ 7 \text { Robert L. Hendrick** } & 1960 \\ 8 \text { Charles Hosler } & 1960 \\ 9 \text { George M. Howe** } & 1960\end{array}$


16 Gordon B. Weir*

17 Joe H. Wolters**

18 Cecil C. Carrier**

19 John C. Capell**

20 Robert C. Copeland**

21 D. Clay McDowell**

22 Albert E. Boyer, Jr.**

23 Harry A. Volkman**

24 Howard M. Frazier**

25 H. Dale Milford**

26 Frank H. Forrester*

27 Leon M. Rottman*

28 James C. Fidler $(\mathrm{H})$

29 William Crawford*

30 Joseph W. Denardo

31 Verl D. Dotson**

32 Charles F. Thomas**

33 Nash C. Roberts, Jr.**

34 Howard E. Reiquam*

35 Norman J. MacDonald**

36 Harry C. Zimmerman**

37 Norman K. Wagner**

38 Robert E. Amsberry**

39 Robert E. Lynott**

40 Howard H. Hanks, Jr.*

41 Roland R. Kessler**

42 George A. Winterling

43 Loren F. Boatman**

44 James E. Smith*

45 Richard D. Goddard

46 Charles W. Stump*

47 Albert W. Duckworth**

48 Clifford H. Watkins**

49 Hubert D. Bagley**

50 George W. Sickels, Jr.**

51 John W. Hambleton**

52 Frank Field**

53 David C. Guild

54 Seth D. Kemble**

55 Conrad L. Johnson**

56 Horace W. Meredith**

57 Leonard M. Slesick**

58 Robert F. Zames**

59 Frederick J. Norman**

60 W. Paul Catoe**

61 John H. Cromwell**

62 James D. Williams**

63 Ronald L. Godbey**

64 Arthur C. Roberts**

65 Boyd E. Quate**

66 Tony Sands**

67 Robert Weaver

68 Jehu D. Ashmore**

69 Robert Kudzma**

70 Charles M. Gertz**

71 Robert E. Wademan*

72 Joel N. Myers**

73 John J. Cahir**

*Deceased $* *$ Inactive
1960

1960

1960

1960

1960

1960

1960

1960

1961

1961

1961

1961

1961

1961

1961

1962

1962

1962

1962

1962

1962

1962

1963

1963

1963

1964

1964

1964

1965

1965

1965

1966

1966

1966

1967

1967

1967

1967

1967

1967

1967

1968

1968

1968

1968

1968

1969

1969

1969

1969

1969

1969

1969

1969
74 John T. Ghiorse, Jr.

1969

75 Richard G. Andrews**

76 Charles M. Umpenhour**

77 William N. Seiler**

78 George R. Wooten, Jr.**

79 Robert T. Ryan

80 Thomas G. Wills

81 Roger L. Triemstra**

82 Elliot Abrams

83 Sidney O. Barnard**

84 William M. Zeliff**

85 Paul S. Joseph

86 Charles Middleton**

87 Douglas B. Cargill**

88 Harry J. Green**

89 Mark E. Eubank

90 Robert E. Zabrecky

91 William S. Thorpe**

92 George R. Fischbeck**

93 John T. McMurray**

94 H. Joe Witte

95 Wayne G. Winston**

96 June Bacon-Bercey**

97 Walton W. Jones

98 Walter A. Lyons**

99 Eric A. Meindl**

100 John F. Beckman

101 Thomas J. Mahoney

102 Mark H. Schumacher**

103 Joseph P. Sobel

104 Allen H. Motew**

105 Virginia J. Bigler-Engler**

106 Jym R. Ganahl

107 Ross J. Dixon, Jr.**

108 William B. Hovey

109 John A. Ebert**

110 Patrick B. Moore**

111 Allan C. Eustis

112 Richard E. Albert

113 Craig J. Woods

114 Winston H. Jervis, Jr.**

115 James J. O'Donnell**

116 William R. Kowal**

117 Norman C. Taylor*

118 Russell G. DuBuc**

119 Thomas E. Skilling III

120 Fred W. Weiss**

121 Robert D. Welti**

122 Russell F. Minshew*

123 Ronald L. Howes

124 Norman F. Lewis**

125 John F. Chandik

126 Kenneth R. Rainey**

127 John R. Bradshaw**

128 Peter F. Giddings

129 Terence F. Kelly

130 Michael R. Smith**

131 Dennis W. Feltgen

132 C. S. Keen**

133 Kenneth B. McCool**
1969

1969

1970

1970

1971

1971

1971

1971

1971

1971

1971

1971

1971

1971

1972

1972

1972

1972

1972

1972

1972

1972

1972

1972

1972

1973

1973

1973

1973

1973

1973

1973

1974

1974

1974

1974

1974

1974

1974

1974

1974

1974

1974

1975

1975

1975

1975

1975

1975

1975

1975

1975

1975

1975

1975

1975

1975

1976

1976

134 John B. Walls**

1976

135 Alan S. Mitleider**

1976

136 David S. Marsh

1976

137 Paul E. Barys

138 James F. Pass**

139 Raymond L. Boylan

1976

1976

1977

140 Joel P. Bartlett, Jr.

141 Robert J. Roseman**

1977

1977

142 Donald S. Noe

1977

143 Richard D. McLaughlin**

144 Don R. Westbrook

145 Charles E. Levy**

146 Howard E. Shapiro

147 Valarie A. Jones**

148 James L. Menard**

149 David P. Murray

150 Donald K. Woods**

151 Richard W. Faurot

152 Jimmie D. Giles

153 Richard J. Katz**

154 Gerald A. Hodak

155 Gary Shore**

156 Roy F. Finden

157 Richard J. Mancini**

158 Gordon D. Rasmussen**

159 Fred J. Jenkins**

160 Richard R. Fletcher

161 Vincent M. Miller**

162 Craig A. Johnson

163 James R. Little

164 Robert P. Soper**

165 Wayne M. Jenkins**

166 Armin J. Ott

167 Dale G. Noah**

168 Jerome B. Taft II

169 Edward J. Ring

170 Theodore F. Fathauer

171 Robert J. Kovachick

172 Robert W. Jacobson, Jr.**

173 Walter Cronise**

174 Michael D. Fairbourne

175 Dallas D. Raines

176 Mike A. Lozano, Jr.

177 James M. Howl 
194 Jeffrey B. Gallant**

195 Keith Eichner

196 Robert W. McLain

197 William D. Kamal

198 Michael H. Graham

199 Arthur E. Hornberger, Jr.

200 Daniel D. Maly

201 Warren D. Nolan

202 Dale N. Zunke

203 Daniel M. Atkinson, Jr.

204 Barry M. Burbank

205 David I. Duisik**

206 Mark S. Russell**

207 C. Michael Rucker

208 Kurt R. Schmitz

209 Harry V. Wappler

210 Paul G. Knight

211 Michael G. Reeves**

212 Robert L. Schwartz**

213 William H. Schubert

214 Wayne C. Shattuck

215 Dennis L. Smith

216 Benjamin D. Gelber

217 Todd Gross

218 Kenneth T. Koch**

219 John J. Malan

220 Jack M. Mercer

221 Glenn E. Schwartz

222 Stanley J. Stachak

223 Joseph A. Dandrea**

224 Thomas L. Magnuson**

225 Joseph C. Conway

226 Bryan S. Norcross

227 Roberta A. Marshment

228 Harvey Leonard

229 James L. Madaus

230 Lawrence J. Mulholland

231 James H. Reif

232 John R. Willing

233 William H. Annen

234 John D. Flanders**

235 James B. Flowers

236 Kevin M. O’Connell**

237 Ronald W. Jackson**

238 Albert L. Roker

239 James W. Bosley

240 Wayne P. Chandler**

241 Daniel G. Dobrowolski

242 Robert E. Gregory

243 Thomas Loffman

244 Nancy A. Chapman

245 Timothy A. Chuey

246 Dennis G. Frary

247 Neil E. Kastor

248 Donald T. McNeely**

249 Michael P. Nelson

250 Donald O. Novak**

251 Eugene M. Rubin
1980

1980

1980

1980

1980

1980

1980

1980

1980

1980

1980

1981

1981

1981

1981

1981

1981

1981

1981

1981

1981

1981

1981

1981

1981

1981

1981

1981

1981

1981

1981

1981

1981

1981

1981

1981

1981

1981

1982

1982

1982

1982

1982

1982

1982

1982

1982

$\overline{* \text { Deceased }} * *$ Inactive

252 Jocelyn K. White**

253 Carey L. Kinsey, Jr.**

254 David K. Towne

255 Richard A. Addis

256 Lawrence D. Hill

257 James G. Duncan

258 Vincent D. Condella, Jr.

259 Glenn N. Burns

260 Louis K. McNally III

261 Douglas P. Kruhoeffer

262 Robert Becker

263 Stuart M. Bowersox**

264 Gregory B. Fishel

265 Anthony O. Johnson

266 Mark L. Nichols**

267 Neal B. Pascal

268 Donald J. Paul

269 Barry A. Richwien

270 Carl Weiss

271 Glenn C. Wood

272 Ronald A. Yaros

274 John G. Bernier

275 Clifford M. Nicholson

276 William E. Kuster**

277 Frank B. Deal, Jr.**

278 John J. Collins

279 John J. Campbell**

280 Thomas E. Hale

281 Robert O. Baron

282 David W. Dahl

283 Willis B. Owings

284 Daniel L. Milham

285 Malcolm P. Sillars**

286 Andre M. Bernier

287 John T. Bielski

288 Edward A. Duranczyk, Jr.

289 William C. Keneely

290 Michael V. Modrick

291 Cyril E. Nefstead

292 Leon F. Pettersen**

293 James J. Schnebelt

294 Mark R. Koontz

295 Donald W. Carson

296 David S. Eiser**

297 Mark Mancuso

298 Randall D. Ollis

299 Thomas C. Rector

300 Terry L. Swails

301 Nicholas J. Gregory

302 Chuck F. Wiese**

303 Barton J. Adrian

304 Raymond J. Ban**

305 Christopher T. Hohmann

306 Gary W. Ley

307 Albert M. Bolton

308 Jim F. Riggs*

309 Tim Ross

310 Donald C. Buser

311 Miles S. Muzio

312 Phil Whitelaw
1982

1982

1982

1982

1982

1982

1982

1982

1982

1982

1982

1982

1982

1982

1982

1982

1982

1982

1982

1982

1982

1982

1982

1982

1982

1982

1982

1982

1982

1982

1982

1982

1982

1983

1983

1983

1983

1983

1983

1983

1983

1983

1983

1983

1983

1983

1983

1983

1983

1983

1983

1983

1983

1983

1983

1983

1983

1983

1983

1983

313 Jeffrey F. Kronschnabel

1983

314 Anthony Cavalier

315 Dale A. Dockus**

1983

316 David N. Eichorn

1983

317 John M. Wendel

318 John C. Dooley**

319 Sally P. Bernier

320 Michael L. Thompson

321 Steven W. Browne

322 Steven L. Horstmeyer

323 Stephen M. Letro**

324 David J. Relihan

325 Paul J. Silvestri**

326 Storm E. Field

327 James E. Brihan**

328 Karen A. Filloon

329 Timothy W. Deegan

330 Harry B. Pringle

331 Jonathan Davies

332 Robert Arnold

333 Donald L. Edwards**

334 Bradford A. Field

335 Valerie Voss

336 John C. Fischer

337 Gail J. Doucet

338 Joseph J. Calhoun

339 Joseph Cioffi

340 Kevin D. Williams

341 David T. Lawyer**

342 Chris D. Thompson

343 Charles S. Herring

344 Troy M. Kimmel, Jr.

345 Jeffrey B. Lawson

346 Donald K. Chilo**

347 Lorraine Stinnett

348 William G. Reh

349 David C. Carlson

350 Paul H. Gross

351 Jeffrey M. Heaton

352 Brian D. Davis

353 David P. Barnes, Jr.

354 David H. Brown

355 Michael H. McClellan

356 John R. Hope

357 Michael J. Hoffman

358 Robert E. Day, Jr.**

359 Rufus R. Hafer**

360 Ron Allen

361 Robert J. Hocks 
373 Dennis J. Hodges**

374 Eric T. DeZubay

375 Paul J. Mroz

376 Matthew Zaffino

377 Janice W. Huff

378 Richard K. Griffin

379 Stephen R. Newman

380 Danny E. Satterfield

381 Norman C. Sebastian

382 Thomas J. Kierein

383 George D. Lessens

384 Joseph Lizura

385 Royal L. Norman, Jr.

386 Duane D. Harding

387 Michael G. Cejka

388 Jay Lennartson**

390 Steven P. Caporizzo

391 William H. Evans

392 Wayne G. Mahar

393 Arnold Rosen

394 Herbert E. Stevens**

395 David M. Miller

396 Wayne C. Hartung

397 Robert G. Lindmeier

398 Daniel W. McCarthy**

399 Thaddeus J. Maguire

400 Andrew L. Weingarten

401 Robert C. Brough

402 Chris S. Edwards**

403 Kenneth A. Schulz

404 Brian A. Albrecht

405 Michael Bono

406 James R. Clarke

407 Andrew A. Avalos

408 Ted W. Keller, Jr.

409 Philip S. Schwarz

410 John W. Adams

411 James R. Wegner**

412 William J. Steffen

413 Lana C. Jones**

414 Sharon L. Graves

415 Shane R. Hollett

416 Donald F. Morelli

417 David A. Carmichael**

418 David C. Whitford**

419 Alan Winfield

420 John M. Wooldridge**

421 David S. Huntress**

422 Tom Lilley**

423 John G. Waunsch

424 Matthew P. Belau

425 Bryan T. Busby

426 Bruce E. Kalinowski

427 Ronald G. Penzkowski

428 Mark B. Rosenthal

429 Sharon L. Akemann

430 Thomas M. Tasselmyer

431 George S. Zabrecky

*Deceased **Inactive
432 Byron K. Webre

434 Holice H. Kaderli, Jr.

435 Glenn E. Johnson

436 Elmer M. Childress

437 Thomas J. Bevacqua**

438 Robert W. Cameron**

439 Keith C. Westerlage

440 James F. Stewart**

441 Andrew C. Provenzano

442 Michael S. Rampy**

443 John C. Gaughan, Jr.

444 Gordon R. Behm

445 Jeffrey T. Jensen

446 William S. Schmidt**

447 Tom Burse

448 Brian H. Durst**

449 Martha L. Austin

450 Bradford T. Huffines

451 Rene J. Brunet III

452 Richard A. Woodford

453 Robert W. Demers**

454 Craig S. Schellsmidt**

455 Paul O. Heppner

456 John R. Boston**

457 John A. Cessarich

458 Theodore E. Textor

459 Daryl W. McCollister

460 Brian T. Alworth

461 Victoria L. Griffin

462 Steven R. Cascione

463 Kenneth A. Aucoin

464 John G. Fausett**

465 David R. Hartman

466 William E. Randby

467 Frederick G. Rixe

468 Philip G. Schreck

469 Martin E. Coniglio

470 James M. Corbin

471 Steve C. LaNore

472 Daniel W. Pope

473 David L. Schaffer**

474 James M. Cantore**

475 Jeffrey D. Klotz

476 Steven C. Bray

477 Declan P. Cannon

478 Frank J. Cariello

479 Patricia L. Cooper**

480 Christopher R. Grote

481 Jon D. Loufman

482 Michael J. Madson

483 Frank J. Marsik

484 Darlene A. Periconi-Balling

485 Phillip P. Stanley**

486 Alan R. Sealls

487 Suzanne S. Knific

488 Jeffrey B. Renner

489 Paul N. Dellegatto

490 David G. Dierks

491 Mark L. Evangelista**
1987

1987

1987

1987

1987

1987

1987

1987

1987

1987

1987

1987

1987

1987

1987

1987

1987

1987

1987

1987

1987

1987

1987

1987

1987

1987

1987

1987

1987

1987

1987

1987

1987

1987

1987

1988

1988

1988

1988

1988

1988

1988

1988

1988

1988

1988

1988

1988

1988

1988

1988

1988

1988

1988

1988

1988

1988

1988

492 James W. Jaggers

1988

493 Ronald A. Hearst II

1988

494 Noreen R. Clark

495 Thomas N. Clark

496 Alan L. Mitchell

497 Robert F. Riggio

498 Charles T. Shutt

Tamara A. Miller-Glahn** 1988

500 George G. Brown 1988

501 Robert H. Rosenzweig 1988

502 Heidi E. Sonen

503 Jeffrey G. Latham**

504 Karroll R. Bohnak

505 Jonathan R. Cash

506 Christopher Curlis**

1988

1988

1988

1988

1988

507 Jeffrey W. Hill

508 Mark J. Scirto

509 Terri A. Smith

510 Michael P. Seidel

511 Phillip B. Williams

512 Robert F. Werner, Jr.

513 James R. Geyer

514 James I. Rasor II

515 Edward I. St. Pe

516 Warren T. Kempf

517 Janice L. Burtt**

518 Kevin M. Carter

519 Linda C. Dolan

520 Samuel M. Evans

521 Hugh A. Kirtek, Jr.

522 Mark D. Lee

523 Mark E. Massaro

524 Cheryl L. Lemke

525 Ronald W. Trenda

526 Anthony J. Derda

527 John J. Guaraldi

528 Tim B. McGill

1988

1988

1988

1988

1988

1988

1988

1988

1988 
552 Kevan W. Ramer

553 Joseph R. Mlodzik

554 Bruce G. Thomas

555 Timothy R. Hedrick

556 Terry Gerbstadt

557 Laura C. York**

558 Travis G. Meyer

559 Robert N. Eisenson

560 Evan A. Rubin

561 Janet E. Pyeatt

562 David A. Hennen

563 Anthony R. Mignone

564 Stephen J. Wohlenhaus

565 Ellen Ferrara

566 Carey T. Coleman

567 Chad E. Myers

568 Terrence P. Casey

569 Brad J. Edwards

570 Michael L. Pass**

571 Mark D. Maggipinto**

572 Joseph R. Calbreath

573 Peter G. J. Parsons

574 Michael F. Maguire

575 Mark D. Wilkinson

576 Lawrence F. Cosgrove

577 Dale L. Jones

578 Michael J. Bracciano

579 Joseph V. Lauria

580 James R. Guy

581 Matthew D. Hinkin

582 Theodore J. Klimasewski

583 Norman D. Dvoskin

584 Daniel S. Connell

585 Jay A. Cardosi

586 Thomas S. Scaman

587 Richard S. Dooley

588 Stephen C. Bellas

589 Edward W. Carroll**

590 Gay H. Dawson**

591 Joan M. Von Ahn

592 David I. Chandley

593 Arthur H. Saxon, Jr.**

594 Ben Woods

595 Joseph R. Diaz

596 Franklin P. Billingsley

597 Cliff Morrison

598 Charles E. Gurney

599 William M. Rowlett

600 Glenn R. Gajownik

601 Connor Vernon

602 Joseph V. DiGiovanni

603 Stephen H. Mumm

604 Bruce M. Avery

605 Robert D. Goosmann

606 Ed C. Matthews

607 Jody L. James**

608 Glen A. Duncan

609 Phillip W. Badgett**

1989

1989

1989

1989

1989

1989

1989

1989

1989

1989

1989

1989

1989

1989

1990

1990

1990

1990

1990

1990

1990

1990

1990

1990

1990

1990

1990

1990

1990

1990

1990

1990

1990

1990

1990

1990

1990

1990

1990

1990

1990

1990

1990

1990

1990

1990

1990

1990

*Deceased **Inactive
610 Elaine B. Lewis

611 Ronald A. Harris

612 Bruce R. Katz

613 Barry A. Brandt

614 Bret M. Atkins

615 Howard I. Bernstein

616 Layne P. Mason

617 William F. Walsh

618 Michael P. Alger

619 Alejandro V. Garcia

620 David M. Kuntz**

621 Jeffrey A. Lazalier

622 Dan P. Meador

623 Robert J. Sopka

624 Donald E. Luehrs, Jr.

625 Richard G. Johnson

626 Jill A. Wiesman**

627 Matthew D. Morano

628 Gary A. Cannalte

629 Tammy A. Garrison

630 Michael K. Glaser**

631 Michele R. Robertson

632 Bradley J. Spakowitz

633 Michael G. Davis

634 James T. Farrell

635 Robert S. Smith

636 John M. Miller

637 Kent Ehrhardt

638 Gary J. Rizzo

639 Gerard J. Tracey

640 Jeffrey G. Hardin

641 William H. Boss

642 W. Marvin Daugherty

643 John W. Robison

644 Stephen M. Udelson

645 Richard T. Marriott

646 Terry L. Kniess

647 Kenneth P. Philips**

648 Mark C. Fuchs

649 Thomas A. Hauf

650 Stephen A. LaPointe

651 William R. Spencer

652 David F. Torchia**

653 Patricia M. Brown

654 J. Curtis Nichols

655 Michael Bigler**

656 John C. Hilgartner

657 Robert W. Peppers

658 Craig A. Weiner

659 Jeffrey Welborn

660 John F. Wheeler

661 James W. Phillips

662 Michael Giunta

663 Patrick A. Core

664 Larry Schick

665 Rick J. Mitchell

666 Dianne Barone

667 Susan A. Mroz**

668 Michael A. Buresh

669 Judson E. Prater, Jr.
1990

670 Vivian L. Brown**

1991

1990

671 Bill Gile

1991

1990

672 Paul T. Huttner

1991

1990

673 David M. Santoro

1991

1990

1990

1990

1990

1990

1990

1990

1990

1990

1990

1990

1990

1990

1990

1990

1990

1990

1990

1990

1990

1991

1991

1991

1991

1991

1991

1991

1991

1991

1991

1991

1991

1991

1991

1991

1991

1991

1991

1991

1991

1991

1991

1991

1991

1991

1991

1991

1991

1991

1991

1991

1991

1991

1991

1991

1991

674 Steve Swienckowski

675 Jesse Walker**

676 Anthony Watts**

677 Joseph C. Goudsward**

678 Michael J. Barsic**

679 Robin J. Reed

680 Terri L. Bennett

681 Zachary R. Berkowitz

682 Michael L. Friedmann

683 Gary M. Lezak

684 Bob Maxon

685 Charles G. Bell

686 Roger E. Vachalek

1991

1991

1991

1991

1991

1991

1992

1992

1992

1992

1992

1992

1992

687 Steven M. Jerve

1992

688 Brian Sussman

689 Christopher M. Wright

690 James Lytle

691 Stephen E. Raleigh

692 Lisa L. Spencer

693 George T. Caldes

694 Alan R. Conklin

695 Mark A. Fox

696 Elissa L. Gruner

697 Michael M. Hernandez

698 Jason P. Laney**

699 Michael E. Kirschvink

700 Michael S. LaPoint

701 Paul E. Bouchereau, Jr.

702 Bob French

703 Robert Perillo 
730 Belinda Jensen

731 Mark Strehl**

1993

732 Harold T. Klingenberg**

733 Michael J. O'Hearn

734 Robert Gregorius

735 David C. Damian

736 Edward T. Piotrowski

737 Scott D. Blalock

738 George H. Kessler, Jr.

739 Robin A. Chisholm

740 Bradley W. Nitz

741 Ronald P. Krasuski

742 John T. Lutzel

743 Barbara Konrad

744 Robert J. Shiels

745 David James

746 Michael J. Latella

747 Scott Mayo

748 Jed R. Castles

749 Adolph M. Andersen, Jr.

750 Sterling S. Poulson

751 Samantha Mohr Aulds

752 Gary Sadowsky

753 Paul L. Williams**

754 David F. Jones

755 Jack Smalley

756 Elisa Robin**

757 Brian Greenwald

758 Evan S. Eldridge

759 David W. Brown

760 Antonio Mastrostefano III

761 Lisa Lucht-Snyder

762 David S. Sweeney

763 Dwight K. Weathers

764 John Jedda**

765 Cory Barron

766 Phillip D. Mann

767 Mary E. Wrobel

768 Mark J. Baden

769 Mark S. Watkins

770 A. Jillene Wahl

771 Jim Barach

772 Kevin H. Goff

773 Linda A. Kozura

774 Stan Boney

775 James D. Aydelott

776 Neal C. Estano

777 John Klevanosky

778 Mark A. Larson

779 Glenn T. Moray

780 David T. Rexroth

781 Steve Teeling

782 Rick E. Katzfey

783 James J. Zahara**

784 Elhanan P. Grigsby III

785 Jeffrey P. Lyons

786 Joseph A. Parise

787 Kathleen A. Orr
788 John Winter

789 James H. Martin

790 David Freeman

791 Mark Finan

792 Mark Allen

793 David L. George, Jr.

794 Kevin L. Orpurt**

795 Anthony J. Pann

796 Jonathan J. Smalstig

797 Theresa L. Varga

798 Ron Roberts

799 Thomas F. Szymanski

800 John Harris

801 Donald P. Harman

802 Richard H. Luterman

803 Christopher P. Whelan

804 Warren J. Madden

805 Stephen J. Svercl

806 Robert James Teske

807 Michael L. Kjar**

808 Julie Broski**

809 Bruce Jones

810 Mel Goldstein

811 Chris Wagenet

812 Dave Vanore

813 Dan Schmit

814 William R. Bakula

815 Veronica Johnson

816 Daniel Bickford

817 Michael A. Brookins

818 Lori A. Miller

819 Thomas G. Sorrells

820 Suzanne Lipsey

821 William R. Korbel

822 Joe Kopecek

823 Rodney L. Hill

824 W. Scott Hall

825 Mike O'Lenick

826 Scott A. Shaw

827 Reg Taylor

828 Arch Kennedy

829 Robert M. Marciano

830 John Scalzi

831 Steve Adamson

832 Neal Barton, Jr.

833 Peter L. Bouchard II**

834 Christopher M. Farrell

835 Craig W. Moeller

836 Jeff Kirk

837 Kevin P. Lemanowicz

838 John C. Honore

839 Thomas Knight

840 David P. Longley

841 Jerry W. Martz

842 Glen Gerberg

843 Edward C. Levy**

844 Camie Stockhausen

845 Charles E. DeBroder

846 Mark E. Johnson

847 Jeffrey A. Tanchak
1994

1994

1994

1994

1994

1994

1994

1994

1994

1994

1994

1994

1994

1994

1994

1994

1994

1994

1994

1994

1994

1994

1994

1994

1994

1994

1994

1994

1994

1994

1994

1994

1994

1994

1994

1994

1994

1994

1994

1994

1995

1995

1995

1995

1995

1995

1995

1995

1995

1995

1995

1995

1995

1995

1995

1995

1995

1995

1995

1995
848 David M. Baker

1995

849 Shea S. Rial

1995

850 Michael C. Morgan**

851 Mark Nelsen

852 Anthony T. Schumacher

853 David A. Fraser**

854 Catherine Horner**

855 Kathy Ellis

856 Jon M. Nese

857 Pete W. Delkus

858 James Spann

859 Austen L. Onek**

860 Mark T. Modrak**

861 Bethany Solomon

862 Terri A. DeBoer

863 Kenneth A. Nagel**

864 Frank Mamola

865 William G. Jacquemin

866 Cecilia Sanvido

867 Matthew A. Lavine

868 Tom Sater

869 Stuart A. Shepard

870 Jeremy Reiner

871 David W. Aguilera

872 David R. Sawyer

873 Ernest G. Fields

874 Marc S. Kramer

875 Daniel W. Lee

876 Michael C. Lowther

877 Lawrence A. Handley

878 Dean R. Wysocki

879 Angelique D. Frame

880 Stephen W. Kersh

881 Richard J. Lawrence

882 Scott Fisher

883 William P. Race

884 Scott Brown

885 John A. Farley

886 Mike Simon

887 Bradley B. Travis

888 Steve W. Weagle

889 David P. Sevcik

890 David Bernard

891 Ron Meritt

892 Steven W. Smith

893 Marcus Lynch

894 Roger M. Twigg

895 Chuck George

896 Keith R. Monahan

897 Mishtu Mukherjee

898 John Marshall

899 Robert S. Van Dillen

900 Donald T. Guthrie

901 Mark L. Bogner

902 Thomas A. Divecchio

903 Mark T. Johnson

904 Harry Johnston

905 Margaret Orr Settoon

906 D. James Siebert

907 Bill Martin

1995

1995

1995

1996

1996

1996

1996

1996

1996

1996

1996

1996

1996

1996

1996

1996

1996

1996

1996

1996

1996

1996

1996

1996

1996

1996

1996

1996

1996

1996

1996

1996

1996

1997

1997

1997

1997

1997

1997

1997

1997

1997

1997

1997

1997

1997

1997

1997

1997 
908 Michael W. Stone

909 Christopher A. Shumway

910 Samuel J. Vincent

911 Matthew J. Chase

912 Mark A. Frankum

913 Daniel H. Thomas
914 Kimberly Adams

915 Todd Fries

916 Patrick J. Griesgraber

917 Ric Kearbey

918 Colin N. Marquis
919 Pamela J. McCown

920 Frederick C. Sandusky

1997

921 Kevin W. Selle

922 Ken Simosko

923 Jon Slater

*Deceased **Inactive

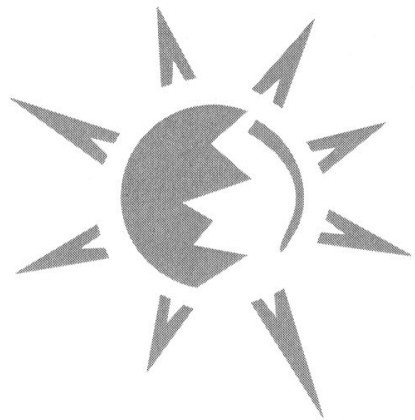

\section{Stochastic Lagrangian Models of Turbulent Diffusion}

Meteorological Monograph No. 48 by Howard C. Rodean

Until now, atmospheric scientists have had to learn the mathematical machinery of the theory of stochastic processes as they went along. With this monograph, atmospheric scientists are given a basic understanding of the physical and mathematical foundations of stochastic Lagrangian models of turbulent diffusion. The culm ination of four years of research, Stochastic Lagrangian Models of Turbulent Diffusion discusses a historical review of Brownian motion and turbulent diffusion; applicable physics of turbulence; definitions of stochastic diffusion; the Fokker-Planck equation; stochastic differential equations for turbulent diffusion; criteria for stochastic models of turbulent diffusion; turbulent diffusion in three dimensions; applications of Thompson's "simplest" solution; ap plication to the convective boundary layer; the boundary condition problem; and a parameterization of turbulence statistics for model inputs.

(C)1996 American Meteorological Society. Hardbound, B\&W, 84 pp., \$55 list $\$ 335$ members (includes shipping and handling). Please send prepaid orders to: Order Department, American Meteorological Society, 45 Beacon St., Boston, MA 02108-3693.

\section{American Meteorological Society}




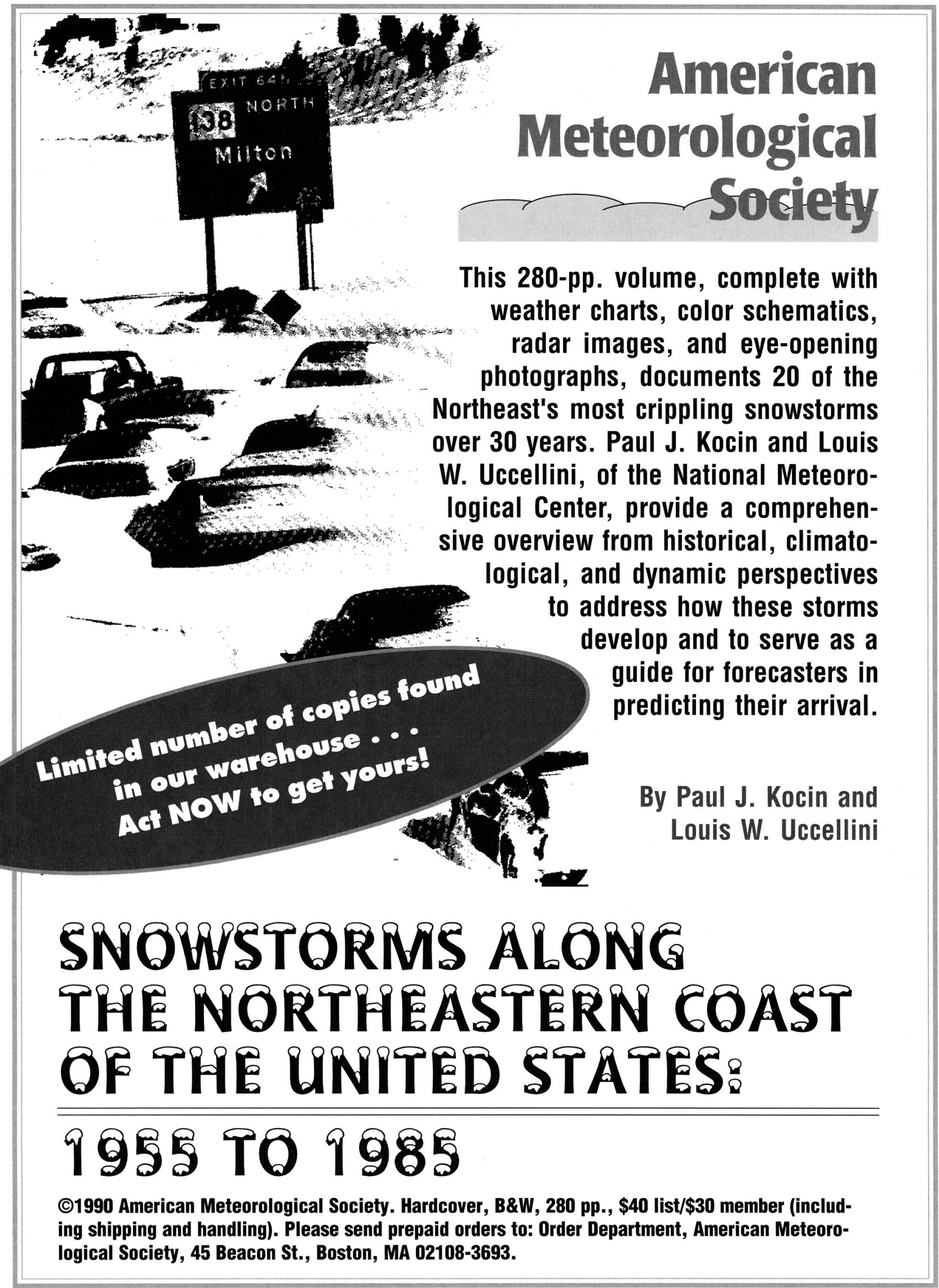

